\title{
FACTORS AFFECTING THE SHEAR BOND STRENGTH OF ORTHODONTIC BRACKETS - A REVIEW OF IN VITRO STUDIES
}

\begin{abstract}
Waleed Bakhadher', Hassan Halawany ${ }^{2,3}$, Nabeel Talic', Nimmi Abraham ${ }^{3}$, Vimal Jacob ${ }^{3}$
Department of Pediatric Dentistry and Orthodontics, College of Dentistry, King Saud University, Riyadh, Saudi Arabia'; Department of Periodontics and Community Dentistry, College of Dentistry, King Saud University, Riyadh, Saudi Arabia ${ }^{2}$; Dental Caries Research Chair, College of Dentistry, King Saud University, Riyadh, Saudi Arabia ${ }^{3}$

Summary: The adhesive material used to bond orthodontic brackets to teeth should neither fail during the treatment period, resulting in treatment delays, untoward expenses or patient inconvenience nor should it damage the enamel on debonding at the end of the treatment. Although the effectiveness of a bonding system and any unfavorable effects on the enamel may be studied by conducting in-vivo studies, it is nearly impossible to independently analyze different variables that influence a specific bonding system in the oral environment. In-vitro studies, on the other hand, may utilize more standardized protocols for testing different bonding systems and materials available. Thus, the present review focused attention on in-vitro studies and made an attempt to discuss material-related, teeth-related (fluorotic vs non-fluorotic teeth) and other miscellaneous factors that influences the shear bond strength of orthodontic brackets. Within the limitations of this review, using conventional acid-etch technique, ceramic brackets and bonding to non-fluorotic teeth was reported to have a positive influence on the shear bond strength of orthodontic brackets, but higher shear bond strength found on using ceramic brackets can be dangerous for the enamel.
\end{abstract}

Keywords: Orthodontic brackets; Acid etching; Bond strength, Fluorosis

\section{Introduction}

Fixed appliance therapy in orthodontics involves bonding brackets to teeth for a period of approximately 2 years. The adhesive material used to bond brackets to teeth should neither fail during the treatment period, resulting in treatment delays, untoward expenses or patient inconvenience nor should it damage the enamel on debonding at the end of the treatment. Although the effectiveness of a bonding system and any unfavorable effects on the enamel may be studied by conducting in-vivo studies, it is nearly impossible to independently analyze different variables that influence a specific bonding system in the oral environment (1). In-vitro studies, on the other hand, may utilize more standardized protocols for testing different bonding systems and materials available. A systematic review and meta-analysis by Finnema et al. (2) had extensively reported the factors affecting in-vitro orthodontic bond strength testing and concluded that the experimental conditions that considerably influence in-vitro bond strength were storage of the bonded specimens in water, photopolymerization time and crosshead speed. Furthermore, the authors also reported that the test conditions were not reported properly in many studies, which could have drastically influenced the outcomes. However, studies evaluating the effect of fluorosis of teeth on the shear bond strength of orthodontic brackets were not included in their systematic review. The present review is an attempt to discuss material-related, teeth-related (fluorotic vs non-fluorotic teeth) and other miscellaneous factors that influences the shear bond strength (SBS) of orthodontic brackets.

\section{Material-related factors}

\subsection{Type of etching material}

A strong bond of composite to enamel was possible ever since the introduction of the use of phosphoric acid in dentistry by Buonocore (3). This procedure, known as acid etching, was later used as a pretreatment technique for bonding orthodontic brackets by Newman (4). Self-etching primers, an alternative for conditioning with phosphoric acid, consists of conventional hydrophilic monomers like Hydroxyethylmethacrylate (HEMA), acidic monomers or acids and have been developed in an attempt to simplify the bonding procedures and minimize procedural errors (5-8). Micro-etching or air abrasion, on the other hand, is a technique in which particles of aluminum oxide $(50 \mu \mathrm{m})$ are propelled against the surface of enamel or another substrate by high air pressure, causing abrasion of the surface. When used, the enamel cuts much faster than dentin due to the loss of energy to a substance's individual resilience 
$(9,10)$. Micromechanical bonding may be obtained by using air abrasion for the preparation of enamel (10). Recently, ultra-short pulsed lasers have been used to prepare teeth prior to orthodontic bonding procedures. These lasers can be focused on the tooth surface with exceptional precision and reproducibility resulting in the ablation of thin layers of enamel without much damage to the adjacent enamel or causing vibration or heating (11).

\subsubsection{Acid etch technique}

Several studies have compared the SBS of orthodontic brackets according to acids used for etching, etchant concentration, duration of etching and variation in etching pattern. Olsen et al. (12) compared the effects, on SBS and bracket failure location, of two adhesives and two enamel conditioners (37\% Phosphoric acid and 10\% Maleic acid). The results showed no significant difference in the mean SBS among the four groups. Carstensen (13) evaluated the effect of different Phosphoric acid concentrations on the SBS of brackets bonded to enamel. The three concentrations examined were $37 \%, 2 \%$ and $5 \%$. This study reported that, the mean SBS after etching with $37 \%$ acid was significantly higher than that after etching with $2 \%$ Phosphoric acid. The effect of etch time and debond interval upon the SBS of metallic orthodontic brackets was studied by Bin Abdullah and Rock (14). The 3 different etching time studied were 15,30 , or 60 seconds and the 3 different debonding time evaluated were 5 or 15 minutes, or 24 hours. The lowest mean SBS was observed in the group of specimens etched for 15 seconds and debonded after 5 minutes. The possible difference in the SBS to acid etched enamel on the different teeth of the dentition was investigated by Hobson et al. (15). The results showed that tooth type had a significant effect on the SBS, with the greatest mean SBS found on the lower first molar teeth and lowest on the upper first molar teeth. Furthermore, the mean SBS was higher on anterior teeth compared to posterior teeth in the upper arch whereas, it was lower on the anterior teeth compared to posterior teeth in the lower arch.

On the other hand, several studies have compared the SBS of orthodontic brackets bonded to teeth after using conventional acid etch technique with that using self-etching primers, air abrasion and lasers for surface preparation.

\subsubsection{Acid etch technique compared to self-etching primers}

Scougall Vilchis et al. (16) compared the SBS of orthodontic brackets bonded with four self-etching adhesives. The authors concluded that, all four self-etching adhesives yielded SBS values higher than the bond strength suggested for routine clinical treatment (5.9 to $7.8 \mathrm{MPa})$. However, the mean SBS value was the highest among the group of teeth treated with the conventional acid etch technique compared to the four tested self-etching adhesive groups. In another study by Scougall Vilchis et al. (17), the conventional acid etch technique reportedly yielded the highest mean SBS value compared to six self-etching primers although the SBS value of all the six self-etching primers were found to be clinically acceptable. Furthermore, the effectiveness of two self-etching primers for bonding brackets were compared with conventional acid etch technique by Vicente et al. (18). The authors reported that no significant differences were observed in the SBS of the three groups evaluated.

Bishara et al. (19) assessed the effects of a fluoride releasing primer compared to that of self-etching primer on the SBS of orthodontic brackets. The authors concluded that, the mean SBS of the fluoride-releasing primer and the self-etching primer was significantly lower than that achieved using conventional acid etch technique. Cehreli et al. (20) assessed and compared the SBS of orthodontic brackets bonded to intact bovine mandibular incisors using four self-etching primer and adhesive formulations, a non-rinse conditioner and acetone adhesive system and a conventional system. The authors concluded that the SBS of the self-etching primer and adhesive systems tested were much lower than that of the conventional acid etch and bond system.

\subsubsection{Acid etch technique compared to air abrasion}

The SBS, location of bond failure and scanning electron microscopic view of the enamel surface preparation was assessed and compared after traditional acid etch technique with an air abrasion surface preparation technique which included two different abrasive particle sizes by Olsen et al. (21). The enamel surface preparation using air abrasion resulted in significantly lower and clinically unacceptable SBS, irrespective of the abrasive particle size, compared to that of using traditional acid etch technique. However, the group of teeth prepared using the larger size abrasive particle $(90 \mu \mathrm{m})$ showed slightly higher SBS values compared to that of smaller size abrasive particle $(50 \mu \mathrm{m})$. Air abrasion without acid etching resulted in significantly lower bond strength and should not be advocated for clinical use (22).

Canay et al. (23) compared the SBS after using conventional acid etch technique using 37\% Phosphoric acid for 15 seconds with that after 1) air abrasion with $50 \mu \mathrm{m}$ Aluminum Oxide; 2) polishing with pumice followed by acid etching; and 3) air abrasion with $50 \mu \mathrm{m}$ Aluminum Oxide followed by acid etching. The results showed that, the air abrasion followed by acid etching group has significantly higher SBS values compared to the other 3 groups whereas air abrasion alone resulted in a significantly lower SBS.

\subsubsection{Acid etching compared to Laser pretreatment}

The SBS after acid etching, laser ablation, acid etching followed by laser ablation and laser ablation followed by acid etching was assessed and compared by Lee et al. (24). The results showed that the mean SBS of the laser group was significantly higher than that of the acid-etched followed 
by laser-ablated group or that of the laser-ablated followed by acid-etched group. However, no significant difference in the mean SBS was observed between laser-ablated group compared to acid-etched group. Alavi et al. (25) compared the SBS and fracture mode of orthodontic brackets bonded to enamel of teeth pretreated with acid etch technique and Er:YAG laser. The results showed that the mean SBS of the laser pretreated group was not significantly different from that of the acid etched group. Sagir et al. (26) compared the effect of laser irradiation at two different pulse settings and acid etching on the SBS of orthodontic brackets. The extracted premolar samples were divided into 3 groups: 1) 37\% Phosphoric acid; 2) Er:YAG laser etching with medium-short pulse mode; and 3) Er:YAG laser etching with quantum-square pulse mode. The results showed that the group pretreated with quantum-square pulse mode Er:YAG laser etching demonstrated significantly higher mean SBS compared to that of the acid-etched group. However, no significant difference was found in the mean SBS values of the two laser groups. Gokcelik et al. (27), on the other hand, compared the SBS of orthodontic brackets after acid etching, laser ablation, self-etching primer application and combined treatment. The results showed no significant difference in the mean SBS of all the groups evaluated.

Oshagh et al. (28) compared the SBS of orthodontic brackets in bonding and rebonding with teeth using $\mathrm{CO}_{2}$ laser versus conventional acid etch technique. The authors concluded that the primary preparation with acid had a higher mean SBS compared to that of $\mathrm{CO}_{2}$ laser. Secondary preparation of the enamel using $\mathrm{CO}_{2}$ laser showed higher mean SBS value than the primary preparation with laser and thus, suggested the use of laser in rebonding of brackets.

The SBS of brackets bonded to teeth after using Er:YAG laser and Ti:Sapphire laser (ultrashort pulsed laser) was evaluated by Lorenzo et al. (12). The extracted premolar teeth included in this study was divided into 3 groups based on the laser treatment performed on the buccal surfaces either as 1) no laser (control); 2) Er:YAG laser; and 3) Ti:Sapphire laser. Each of these 3 groups was further divided into 2 subgroups based on whether $37 \%$ Orthophosphoric acid etching was performed after laser treatment or not. The results showed that, in the non-acid etched teeth samples, the mean SBS values of those teeth treated with Ti:Sapphire laser was significantly higher than those treated with Er:YAG laser or the control group. Furthermore, acid etching had no significant effect on the SBS after treating with Ti:Sapphire laser.

\subsection{Types of brackets}

The SBS of stainless steel (SS) and ceramic brackets bonded using chemically and light-cured composite resins were evaluated by Joseph and Rossouw (29). Brackets were bonded to 48 canine teeth after acid etching and were divided into 4 groups: 1) SS brackets and chemically cured resin; 2) ceramic brackets and chemically cured resin; 3) ceramic brackets and light-cured resin; and 4) SS brack- ets and light-cured resin. The authors concluded that, SBS greater than that clinically acceptable were produced by all combinations. Furthermore, the ceramic group showed a significantly higher SBS than that of the SS group. Enamel fractures occurred in $40 \%$ of the group of ceramic brackets and chemically cured resin. The fracture of enamel is a real possibility during therapy or at debonding of the ceramic brackets.

The mean SBS of polycrystalline ceramic brackets were found to be significantly higher than that of SS brackets in several other studies (30-32). However, one study (32) reported that the mean SBS value was found to be lowest among single crystal ceramic brackets. Liu et al. (33) evaluated and compared the SBS of a collapsible monocrystalline bracket (Inspire, Ormco, Orange, Calif) with that of another collapsible ceramic bracket (Clarity, 3M Unitek, Monrovia, Calif) and a metal bracket. Two orthodontic adhesives were used to bond these brackets. The results showed no significant differences between the different combinations of brackets and adhesives.

The SBS of ceramic bracket (Transcend 6000, 3M Unitek) was measured and compared with that of a metal-reinforced ceramic bracket (Clarity, 3M Unitek) by Mundstock et al. (34). The authors reported that the mean SBS of Transcend 6000 was $21.19 \pm 5.94 \mathrm{MPa}$ whereas, that of metal-reinforced ceramic bracket was $13.27 \pm 5.4$ $\mathrm{MPa}$, which was above the accepted minimal SBS range (5.9 to $7.8 \mathrm{MPa}$ ) for successful clinical bonding suggested by Reynolds (35).

The SBS of plastic brackets was evaluated by Guan et al. (36) and was compared to that of conventional metal brackets. The results indicated that, the SBS of the 4 brands of plastic brackets tested was significantly lower than that of the conventional metal brackets.

\subsection{Bracket base design and size}

Sorel et al. (37) evaluated the SBS of metallic orthodontic brackets with a laser structured base (Discovery, Dentaurum, Ispringen, Germany) and compared it with a standard system with a simple foil mesh base (Minitrimm, Dentaurum). The results revealed that the mean SBS was significantly higher for the brackets with laser structured base compared to that of the brackets with foil mesh base. In addition, the effect of orthodontic bracket base design on mean SBS was studied by Sharma-Sayal et al. (2003). The design of the base of brackets tested were: 1) 60-gauge, microetched foil-mesh base; 2) machined, integral, microetched base with mechanical undercuts; 3) 80-gauge foil-mesh base; 4) 80-gauge layered onto 150-gauge, microetched foil-mesh base; 5) 100-gauge microetched foil-mesh base; and 6) injection molded, 100-gauge, microetched foil-mesh base. The results showed that the 60-gauge microetched foil-mesh base brackets had the highest mean SBS at 1 hour. The SBS of different brands of metal orthodontic brackets were assessed and compared by Cozza et al. (38). The 5 brands tested were: 1) Victory Se- 
ries (3M Unitek, Monrovia, Calif); 2) Mini Dyna-Lock (3M Unitek); 3) Mini Sprint (Forestadent, Pforzheim, Germany); 4) Topic (Dentaurium, Inspringen, Germany) and 5) Equilibrium 2 (Dentaurium). The results showed that, Equilibrium 2 demonstrated a significantly higher mean SBS compared to other brands except Victory Series.

Another study (39) evaluated the influence of 6 different types of metal interlock brackets of different sizes and with different base designs on the SBS. The bracket base designs and sizes tested were: 1) retention groove base (Dyna-Lock, Unitek, Monrovia, Calif); 2) circular concave base (Accurarch appliance Formula-R, Tomy, Tokyo, Japan); 3) double mesh with $5.1 \times 10^{-2} \mathrm{~mm}^{2}$ mesh size (Ultratrimm, Dentaurum, Ispringen, Germany); 4) double mesh, $3.1 \times 10^{-2} \mathrm{~mm}^{2}$ (Minidiagonali Roth, Leone, Florence, Italy); 5) double mesh, $3.1 \times 10^{-2} \mathrm{~mm}^{2}$ (Tip-edge Rx-I, TP Orthodontics, LaPorte, Ind; and 6) double mesh, $2.9 \times 10^{-2} \mathrm{~mm}^{2}$ (Mini Diamond, Ormco, Glendora, Calif). The results showed that, the brackets with circular concave base (Tomy) demonstrated higher mean SBS than mesh-based brackets. Furthermore, among the brackets with mesh base, the brackets with the larger mesh size showed higher mean SBS compared to those with smaller mesh size.

\subsection{Adhesives}

Bishara et al. (5) evaluated and compared the SBS of orthodontic brackets bonded using composite, glass ionomer and adhesive primer adhesive systems. The brackets were bonded by using a glass ionomer adhesive after using a 20\% Polyacrylic acid enamel conditioner, a composite resin adhesive after etching the enamel using 37\% Phosphoric acid or the same composite resin adhesive after using an acidic primer. The results showed that the mean SBS of the composite resin-Phosphoric acid adhesive system was significantly higher than the other systems tested whereas the mean SBS of the glass ionomer adhesive system was significantly lower compared to the other systems. Furthermore, the acid primer used with composite resin adhesive demonstrated the least mean SBS. The SBS of a light-cured, resin-reinforced glass ionomer (Fuji Ortho LC) and a composite adhesive combined with a self-etching primer (Transbond XT and Transbond Plus) was evaluated and compared after different setting times ( 5 minutes and 15 minutes) by Movahhed et al. (40). The results showed that the mean SBS was higher with Transbond XT than with Fuji Ortho LC after both setting times.

\subsection{Bonding to restorative materials}

Bonding orthodontic brackets to amalgam surface was studied by Sperber et al. (41). One hundred and eight standardized amalgam cylinder samples were divided into 9 groups based on surface treatment technique and resin type. The amalgam surfaces were either polished, sandblasted using $50 \mu \mathrm{m}$ aluminum oxide, or chemically corroded and
SPEED brackets (Strite Industries, Cambridge, Ontario) were bonded onto these prepared surfaces using Phase II (Reliance Orthodontic Products Inc, Itasca, Ill), or C \& B Metabond (Parkell, Farmingdale, NY) adhesives. All the resin systems showed significantly high SBS on sandblasted amalgam surface. The authors concluded that laboratory acceptable SBS of orthodontic brackets to amalgam was possible.

The SBS of SS brackets bonded to different ceramic surfaces was investigated by Abu Alhaija and Al-Wahadni (42). Standard edgewise metal premolar brackets were bonded to In-Ceram, IPS-Empress and conventional metal ceramic crowns. The results showed that the IPS-Empress group showed significantly low mean SBS compared to metal ceramic and In-Ceram groups.

Rambhia et al. (43) assessed the SBS of orthodontic brackets bonded to provisional crown materials utilizing two different adhesives. Forty cylindrical specimens were prepared from provisional crown materials which included Integrity, Jet, Protemp and Snap. Two brands of orthodontic brackets, Clarity or Victory, were bonded to ten specimens from each of the provisional crown material using either Fuji Ortho LC or Ortho Bracket Adhesive. The results showed that the mean SBS of brackets bonded to Snap was significantly low compared to the other materials. However, no significant difference was observed between the two brackets or the two adhesives used. Furthermore, the bond failure for all the specimens was found to occur between the provisional crown material and the adhesive resin.

The combined effects of material type, surface treatment, and thermocycling on the SBS of orthodontic brackets bonded to provisional crown materials was evaluated by $\mathrm{Al}$ Jabbari et al. (44). Sixty cylindrical specimens were prepared from each of the provisional crown material which included ProTemp, Trim Plus, Trim II, and Superpont $\mathrm{C}+\mathrm{B}$, and were divided into three groups according to the following pre-treatments: 1) ground with silica carbide paper; 2) polished with pumice and 3) sandblasted with $50 \mu \mathrm{m}$ aluminum oxide. Transbond XT light-cured composite resin was used to bond SS brackets (Victory Series, 3M) to the specimens. After bonding the brackets, half of the specimens from each group were thermocycled 500 times in $5^{\circ} \mathrm{C}$ and $55^{\circ} \mathrm{C}$ water baths. The results showed significant differences in the SBS among the provisional materials, surface treatments and thermocycling. The authors concluded that, the provisional material type, surface treatment and artificial aging exerted a significant effect on the SBS of brackets. The authors also stated that sandblasting exerted a beneficial effect on the SBS.

\section{Teeth-related factors}

\subsection{Fluorosis}

Although the enamel crystals in severely fluorosed teeth may be separated by larger inter-rod spaces, no other significant difference in the enamel crystals were observed 
compared to non-fluorosed teeth (45). Consequently, no relationship between etching pattern and the severity of fluorosis has been observed in previous studies $(46,47)$. However, several studies reported that fluorosis had a negative influence on the SBS of orthodontic brackets. Adanir et al. (48) evaluated the effect of fluorosis on the SBS of orthodontic brackets. Thirty fluorosed teeth, selected according to the modified Thystrup and Fejerskov index and 15 non-fluorosed teeth were included in the study. The results showed that fluorosis significantly reduced the SBS of orthodontic brackets.

Gungor et al. (49) evaluated the effects of fluorosis and self-etching primers on the SBS of orthodontic brackets. Twenty four fluorosed teeth selected according to the modified Thylstrup and Fejerskov index and 24 non-fluorosed extracted human premolar teeth were randomly assigned to 4 groups of 12 each. Standard etching protocol was followed and brackets were bonded with Light Bond in group I (nonfluorosed teeth) and II (fluorosed teeth). Similarly, Transbond Plus self-etching primer was used and brackets were bonded using Transbond XT Light Cure Adhesive in groups III (nonfluorosed teeth) and IV (fluorosed teeth). The results showed that the mean SBS in group II (Light Bond + fluorosis) was significantly lower than that of the other groups. The authors concluded that enamel fluorosis significantly decreased the SBS of orthodontic brackets when standard etching protocol was used. However, the authors reported that a satisfactory SBS was obtained when self-etching primer was used for bonding brackets to fluorosed teeth. Another study (50) compared the SBS of orthodontic brackets bonded to fluorosed and non-fluorosed teeth with self-etching primer and Phosphoric acid. Forty mildly fluorosed teeth selected according to Thylstrup and Fejerskov index (1-3; mild fluorosis) and 40 non-fluorosed teeth were randomly divided into two subgroups according to the pre-treatment: 1) $37 \%$ Phosphoric acid applied for 30 seconds; 2) self-etching primer (Transbond Plus). The brackets were bonded using Transbond XT, cured for 20 seconds and the SBS measured after 1000 thermocycles. No difference in the SBS was observed between mildly fluorosed and non-fluorosed teeth etched with $37 \%$ Phosphoric acid for 30 seconds. However, the mean SBS of orthodontic brackets bonded to mildly fluorosed teeth treated with self-etching primer showed lower values compared to the non-fluorosed teeth.

Suma et al. (51) evaluated the effect of air abrasion on the SBS of brackets bonded to fluorosed teeth. Sixty extracted premolar teeth with moderate to severe fluorosis according to Dean's criteria were divided into 3 groups: 1) acid etching followed by bonding brackets with Tranbond XT; 2) air abrasion followed by acid etching after which brackets were bonded using Transbond XT; and 3) air abrasion followed by acid etching after which brackets were bonded using Enlight LC. The authors concluded that, irrespective of the bonding material used, air abrasion followed by acid etching showed significantly higher mean SBS compared to acid etching alone.

\section{Miscellaneous factors}

Some studies have investigated the influence of mouthwashes and other materials used for caries prevention/ enamel remineralization on the SBS of orthodontic brackets. Bishara et al. (52) assessed the effect of application of chlorhexidine on the SBS of orthodontic brackets. The authors concluded that, chlorhexidine either applied over the bracket and tooth surfaces after the bonding procedure was completed or applied as a prophylactic paste over the unetched enamel surface before the bonding procedure was initiated, had no significant effect on the SBS of orthodontic brackets.

On the other hand, Kecik et al. (53) evaluated the effect of Casein Phosphopeptide-Amorphous Calcium Phosphate (CCP-ACP) and Acidulated Phosphate Fluoride (APF) on the SBS of orthodontic brackets. The authors concluded that CPP-ACP, either alone or combined with APF, had no significant effect on the SBS of orthodontic brackets. Al-Kawari and Al-Jobair (54) investigated the effect of CPP-ACP, fluoride-containing CPP-ACP and 5\% Sodium fluoride on the SBS of orthodontic brackets before compared to that after acid-etching. The authors concluded that, when fluoride-containing CPP-ACP was applied after acid-etching, the SBS of orthodontic brackets was significantly increased.

\section{Conclusion}

Our literature review revealed that both material- and teeth-related factors influenced the SBS of orthodontic brackets. However, this cannot be considered as a comprehensive review in view of the fact that it has not included all the material-related, teeth-related and other miscellaneous factors that may have direct or indirect influence on the SBS of orthodontic brackets. Within its limitations, using conventional acid-etch technique, ceramic brackets and bonding to non-fluorotic teeth was reported to have a positive influence on the SBS of orthodontic brackets, but higher shear bond strength found on using ceramic brackets can be dangerous for the enamel. More research is required to develop our understanding of the role of these factors in influencing the shear bond strength of orthodontic brackets.

\section{References}

1. Leloup G, D'Hoore W, Bouter D, Degrange M, Vreven J. Meta-analytical review of factors involved in dentin adherence. J Dent Res 2001 Jul; 80(7): 1605-14.

2. Finnema KJ, Ozcan M, Post WJ, Ren Y, Dijkstra PU. In-vitro orthodontic bond strength testing: a systematic review and meta-analysis. Am J Orthod Dentofacial Orthop 2010 May; 137(5): 615-22 e3.

3. Buonocore MG. A simple method of increasing the adhesion of acrylic filling materials to enamel surfaces. J Dent Res 1955 Dec; 34(6): 849-53.

4. Newman GV. Epoxy adhesives for orthodontic attachments: progress report. Am J Orthod 1965 Dec; 51(12): 901-12.

5. Bishara SE, Gordan VV, VonWald L, Jakobsen JR. Shear bond strength of composite, glass ionomer, and acidic primer adhesive systems. Am J Orthod Dentofacial Orthop 1999 Jan; 115(1): 24-8.

6. Fritz UB, Finger WJ. Bonding efficiency of single-bottle enamel/dentin adhesives. Am J Dent 1999 Dec; 12(6): 277-82.

7. Perdigao J, Lopes L, Lambrechts P, Leitao J, Van Meerbeek B, Vanherle G. Effects of a self-etching primer on enamel shear bond strengths and SEM morphology. Am J Dent 1997 Jun; 10(3): 141-6. 
8. Sano H, Yoshikawa T, Pereira PN, et al. Long-term durability of dentin bonds made with a self-etching primer, in vivo. J Dent Res 1999 Apr; 78(4): 906-11.

9. Gerbo LR, Barnes CM, Leinfelder KF. Applications of the air-powder polisher in clinical orthodontics. Am J Orthod Dentofacial Orthop 1993 Jan; 103(1): 71-3.

10. Goldstein RE, Parkins FM. Air-abrasive technology: its new role in restorative dentistry. J Am Dent Assoc 1994 May; 125(5): 551-7.

11. Lorenzo MC, Portillo M, Moreno P, et al. In vitro analysis of femtosecond lase as an alternative to acid etching for achieving suitable bond strength of brackets to human enamel. Lasers Med Sci 2014 May; 29(3): 897-905.

12. Olsen ME, Bishara SE, Damon P, Jakobsen JR. Evaluation of Scotchbond Multipurpose and maleic acid as alternative methods of bonding orthodontic brackets Am J Orthod Dentofacial Orthop 1997 May; 111(5): 498-501.

13. Carstensen W. Effect of reduction of phosphoric acid concentration on the shear bond strength of brackets. Am J Orthod Dentofacial Orthop 1995 Sep; 108(3): 274-7.

14. Bin Abdullah M, Rock WP. The effect of etch time and debond interval upon the shear bond strength of metallic orthodontic brackets. British journal of orthodontics 1996; 23(2): 121-4

15. Hobson RS, McCabe JF, Hogg SD. Bond strength to surface enamel for different tooth types. Dent Mater 2001 Mar; 17(2): 184-9.

16. Scougall Vilchis RJ, Yamamoto S, Kitai N, Yamamoto K. Shear bond strength of orthodontic brackets bonded with different self-etching adhesives. Am J Orthod Dentofacial Orthop 2009 Sep; 136(3): 425-30.

17. Scougall-Vilchis RJ, Ohashi S, Yamamoto K. Effects of 6 self-etching primers on shear bond strength of orthodontic brackets. Am J Orthod Dentofacial Orthop 2009 Apr; 135(4): 424 e1-7; discussion 5.

18. Vicente A, Bravo LA, Romero M, Ortiz AJ, Canteras M. Shear bond strength of orthodontic brackets bonded with self-etching primers. Am J Dent 2005 Aug, 18(4): 256-60

19. Bishara SE, Ajlouni R, Laffoon JF, Warren JJ. Effect of a fluoride-releasing selfetch acidic primer on the shear bond strength of orthodontic brackets. Angle Orthod 2002 Jun; 72(3): 199-202.

20. Cehreli ZC, Kecik D, Kocadereli I. Effect of self-etching primer and adhesive formulations on the shear bond strength of orthodontic brackets. Am J Orthod Dentofacial Orthop 2005 May; 127(5): 573-9; quiz 625-6.

21. Olsen ME, Bishara SE, Damon P, Jakobsen JR. Comparison of shear bond strength and surface structure between conventional acid etching and air-abrasion of human enamel. Am J Orthod Dentofacial Orthop 1997 Nov; 112(5): 502-6.

22. Abu Alhaija ES, Al-Wahadni AM. Evaluation of shear bond strength with different enamel pre-treatments. Eur J Orthod 2004 Apr; 26(2): 179-84.

23. Canay S, Kocadereli I, Akça E. The effect of enamel air abrasion on the retention of bonded metallic orthodontic brackets. Am J Orthod Dentofacial Orthop 2000 Jan; 117(1): 15-9.

24. Lee BS, Hsieh TT, Lee YL, Lan WH, Hsu YJ, Wen PH, et al. Bond strengths of orthodontic bracket after acid-etched, Er:YAG laser-irradiated and combined treatment on enamel surface. Angle Orthod 2003 Oct; 73(5): 565-70.

25. Alavi S, Birang R, Hajizadeh F. Shear bond strength of orthodontic brackets after acid-etched and erbium-doped yttrium aluminum garnet laser-etched. Dent Res J (Isfahan) 2014 May; 11(3): 321-6.

26. Sagir S, Usumez A, Ademci E, Usumez S. Effect of enamel laser irradiation at different pulse settings on shear bond strength of orthodontic brackets. Angle Orthod 2013 Nov; 83(6): 973-80.

27. Gokcelik A, Ozel Y, Ozel E, et al. The influence of Er:YAG laser conditioning versus self-etching adhesives with acid etching on the shear bond strength of orthodontic brackets. Photomed Laser Surg 2007 Dec; 25(6): 508-12.

28. Oshagh M, Pakshir HR, Najafi HZ, Naseri MM, Nasrabadi NI, Torkan S. Comparison of the shear bond strength of orthodontic brackets in bonding and rebonding: preparation with laser versus conventional acid etch technique. Photomed Laser Surg 2013 Aug; 31(8): 360-4.

29. Joseph VP, Rossouw E. The shear bond strengths of stainless steel and ceramic brackets used with chemically and light-activated composite resins. Am J Orthod Dentofacial Orthop 1990 Feb; 97(2): 121-5.

30. Forsberg CM, Hagberg C. Shear bond strength of ceramic brackets with chemical or mechanical retention. Br J Orthod 1992 Aug; 19(3): 183-9.
31. Franklin S, Garcia-Godoy F. Shear bond strengths and effects on enamel of two ceramic brackets. J Clin Orthod 1993 Feb; 27(2): 83-8.

32. Viazis AD, Cavanaugh G, Bevis RR. Bond strength of ceramic brackets under shear stress: an in vitro report. Am J Orthod Dentofacial Orthop 1990 Sep; 98(3): 214-21

33. Liu JK, Chung CH, Chang CY, Shieh DB. Bond strength and debonding characteristics of a new ceramic bracket. Am J Orthod Dentofacial Orthop 2005 Dec; 128(6): 761-5; quiz 802

34. Mundstock KS, Sadowsky PL, Lacefield W, Bae S. An in vitro evaluation of a metal reinforced orthodontic ceramic bracket. Am J Orthod Dentofacial Orthop 1999 Dec; 116(6): 635-41.

35. Reynolds I. A review of direct orthodontic bonding. Br J Orthodont $1975 ; 2: 171-8$.

36. Guan G, Takano-Yamamoto T, Miyamoto M, Hattori T, Ishikawa K, Suzuki K. Shear bond strengths of orthodontic plastic brackets. Am J Orthod Dentofacial Orthop 2000 Apr; 117(4): 438-43.

37. Sorel O, El Alam R, Chagneau F, Cathelineau G. Comparison of bond strength between simple foil mesh and laser-structured base retention brackets. Am J Orthod Dentofacial Orthop 2002 Sep; 122(3): 260-6.

38. Cozza P, Martucci L, De Toffol L, Penco SI. Shear bond strength of metal brackets on enamel. Angle Orthod $2006 \mathrm{Sep}$; 76(5): 851-6.

39. Wang WN, Li CH, Chou TH, Wang DD, Lin LH, Lin CT. Bond strength of various bracket base designs. Am J Orthod Dentofacial Orthop 2004 Jan; 125(1): 65-70.

40. Movahhed HZ, Ogaard B, Syverud M. An in vitro comparison of the shear bond strength of a resin-reinforced glass ionomer cement and a composite adhesive for bonding orthodontic brackets. Eur J Orthod 2005 Oct; 27(5): 477-83.

41. Sperber RL, Watson PA, Rossouw PE, Sectakof PA. Adhesion of bonded orthodontic attachments to dental amalgam: In vitro study. Am J Orthod Dentofacial Orthop 1999 Nov; 116(5): 506-13

42. Abu Alhaija ES, Al-Wahadni AM. Shear bond strength of orthodontic brackets bonded to different ceramic surfaces. Eur J Orthod 2007 Aug; 29(4): 386-9.

43. Rambhia S, Heshmati R, Dhuru V, Iacopino A. Shear bond strength of orthodontic brackets bonded to provisional crown materials utilizing two different adhesives. Angle Orthod 2009 Jul; 79(4): 784-9.

44. Al Jabbari YS, Al Taweel SM, Al Rifaiy M, Alqahtani MQ, Koutsoukis T, Zinelis S. Effects of surface treatment and artificial aging on the shear bond strength of orthodontic brackets bonded to four different provisional restorations. Angle Orthod 2014 Jul; 84(4): 649-55.

45. Fejerskov O, Manji F, Baelum V. The nature and mechanisms of dental fluorosis in man. Journal of dental research 1990; 69 Spec No: 692-700; discussion 21.

46. Al-Sugair MH, Akpata ES. Effect of fluorosis on etching of human enamel. J Oral Rehabil 1999 Jun; 26(6): 521-8.

47. Ng'ang'a PM, Ogaard B, Cruz R, Chindia ML, Aasrum E. Tensile strength of orthodontic brackets bonded directly to fluorotic and nonfluorotic teeth: an in vitro comparative study. Am J Orthod Dentofacial Orthop 1992 Sep; 102(3): 244-50.

48. Adanir N, Turkkahraman H, Yalcin Gungor A. Effects of adhesion promoters on the shear bond strengths of orthodontic brackets to fluorosed enamel. Eur J Orthod 2009 Jun; 31(3): 276-80.

49. Gungor AY, Turkkahraman $\mathrm{H}$, Adanir N, Alkis $\mathrm{H}$. Effects of fluorosis and self etching primers on shear bond strengths of orthodontic brackets. Eur J Dent 2009 Jul; 3(3): 173-7.

50. Isci D, Sahin Saglam AM, Alkis H, Elekdag-Turk S, Turk T. Effects of fluorosis on the shear bond strength of orthodontic brackets bonded with a self-etching primer. Eur J Orthod 2011 Apr; 33(2): 161-6.

51. Suma S, Anita G, Chandra Shekar BR, Kallury A. The effect of air abrasion on the retention of metallic brackets bonded to fluorosed enamel surface. Indian J Dent Res 2012 Mar-Apr; 23(2): 230-5.

52. Bishara SE, Vonwald L, Zamtua J, Damon PL. Effects of various methods of chlorhexidine application on shear bond strength. Am J Orthod Dentofacial Orthop 1998 Aug; 114(2): 150-3.

53. Kecik D, Cehreli SB, Sar C, Unver B. Effect of acidulated phosphate fluoride and casein phosphopeptide-amorphous calcium phosphate application on shear bond strength of orthodontic brackets. Angle Orthod 2008 Jan; 78(1): 129-33.

54. Al-Kawari HM, Al-Jobair AM. Effect of different preventive agents on bracket shear bond strength: in vitro study. BMC Oral Health 2014; 14: 28

Received: $26 / 05 / 2015$

Accepted in revised form: 13/06/2015

\section{Corresponding author:}

Dr. Hassan Halawany, Dental Caries Research Chair, College of Dentistry, King Saud University, P.O. Box 60169, Riyadh 11545, Saudi Arabia; e-mail: halawanyhksu@gmail.com 